\title{
Personzentriertes diagnostisches Verstehen und der ganzheitliche Blick auf das Individuum
}

\author{
Marieluise Brückl
}

Online publiziert: 9. Juni 2020

(c) Der/die Autor(en) 2020

\begin{abstract}
Zusammenfassung Im Personzentrierten Ansatz wird der Mensch in seiner Gesamtheit betrachtet und die Ursache für Störungen in der Konstellation der Selbststruktur angenommen. In der therapeutischen Arbeit mit Jugendlichen gilt es zu verstehen, welche der vielfältigen Erfahrungen in das Selbstkonzept vollständig integriert werden konnten und welche vielleicht nur verzerrt aufgenommen oder ganz verleugnet werden mussten. Diagnostik im personzentrierten Verständnis ist ein Teil der Therapie bzw. eine den Prozess begleitende Diagnostik und wird an Hand der Arbeit mit einer fünfzehnjährigen Jugendlichen beschrieben.
\end{abstract}

Schlüsselwörter Personzentrierte Diagnostik . Jugendlichenpsychotherapie · Depression • Selbstkonzept · Inkongruenz

\section{Person-centered diagnostic understanding and} a holistic view of the individual

Summary In the person-centred approach people are seen in their entirety and the reason of disorders is believed in the constellation of selfstructure. In the therapeutic work with young patients it is important to unterstand which of the diverse experiences could be fully integrated into the selfconcept or which ones had to be distorted or totally denied. Diagnosis in a person-centred sense of view is part of therapy or a process concomitant with therapy. This will be described based on the work with a 15 year old girl.

M. Brückl ( $\varangle)$

Hernalser Hauptstraße 79a/2/36, 1170 Wien, Österreich marieluise.brueckl@gmail.com

\section{Einleitung}

Der Personzentrierte Ansatz ist in seiner Ausrichtung eine Abwendung von der pathologischen Perspektive hin zu einer entwicklungsfördernden. Im Vordergrund der Personzentrierten Kinder- und Jugendlichenpsychotherapie steht somit die ganzheitliche Entwicklung und nicht der bloße Abbau von Symptomen. Im folgenden Artikel wird der diagnostische Prozess und wie dieser in die therapeutische Arbeit eingebunden ist an Hand der Arbeit mit einer jugendlichen Klientin (ich nenne sie hier Lena) dargestellt.

Die Personzentrierte Kinder- und Jugendlichenpsychotherapie hat in den letzten Jahrzehnten im deutschsprachigen Raum eine Weiterentwicklung und inhaltliche Differenzierung erfahren und weist mittlerweile eine Vielzahl an Veröffentlichungen und Praxisberichten auf. $\mathrm{Zu}$ den unterschiedlichsten Störungsbildern und psychosozialen Problemlagen wurde zum Beispiel von Behr et al. (2009) der Sammelband „Psychotherapie mit Kindern und Jugendlichen. Personzentrierte Methoden und interaktionelle Behandlungskonzepte" herausgegeben. Dem Einsatz der Kinder- und Jugendlichenpsychotherapie in unterschiedlichen Settings haben sich Weinberger und Papastefanou (2008) in ihrem Buch „Wege durchs Labyrinth. Personzentrierte Beratung und Psychotherapie mit Jugendlichen" gewidmet. Unbedingt erwähnenswert ist auch die Publikation der Handlungsleitlinien (Behr et al. 2012) zu unterschiedlichen Störungsbildern, die von verschiedenen Autorengruppen verfasst wurden. Darin finden sich neben der Klassifikation der Symptome nach ICD-10 konkrete Hinweise zur methodenspezifischen Störungsentstehung und zu einer umfassenden und differenzierten Diagnostik in der Personzentrierten Kinder- und Jugendlichenpsychotherapie. 
Schon Carl R. Rogers, der Begründer der Personzentrierten Psychotherapie, hat sich in den Anfängen seiner beruflichen Tätigkeit und am Beginn seiner Konzeptentwicklung mit der Entwicklung und Begleitung von Kindern und ihren Bezugspersonen auseinandergesetzt und in seinem Buch „The Clinical Treatment of The Problem Child“ von 1939 die Grundlage für die heutige therapeutische personzentrierte Arbeit mit Kindern und Jugendlichen gelegt (vgl. Reisel 2014). Zwar hat sich Rogers gegen Statusdiagnosen ausgesprochen, nicht aber gegen einen diagnostischen Prozess an sich. Vielmehr spricht er von der Bedeutung prozessualer Diagnostik im Rahmen der Psychotherapie: „In einem sehr bedeutungsvollen und genauen Sinn ist die Therapie Diagnose, und diese Diagnose ist ein Prozeß, der eher in der Erfahrung des Klienten abläuft als im Intellekt des Klinikers“ (Rogers 2003, S. 208).

Am Beginn der personzentrierten Arbeit mit Kindern und Jugendlichen bedeutet Diagnostik zunächst das Erfassen der Ist-Situation (vgl. Jürgens-Jahnert 2002), also jener Situation, die zu der Entscheidung geführt hat eine Psychotherapeutin aufzusuchen (siehe Therapieeingangsdiagnostik unten). Jürgens-Jahnert (2002) liefert eine sehr ausführliche Beschreibung der Eingangsdiagnostik und wie diese in den weiteren Behandlungsverlauf einfließt. Das „auf dem Boden einer übergreifenden klientenzentrierten Krankheitslehre gewonnene Wissen um die fallspezifischen Störungsursachen bildet dann eine entscheidende Grundlage für die Planung und Durchführung der individuellen Behandlung" (Jürgens-Jahnert 2002, S. 262). Zu dieser Grundlage gehört sowohl die Erstellung einer Statusdiagnose nach ICD-10 als auch die Beachtung der unten beschriebenen Aspekte der personzentrierten prozessualen Diagnostik.

Die in der ICD-10 phänomenologisch beschriebenen Symptome der zugrunde liegenden psychischen Störung werden im Personzentrierten Ansatz als Ausdruck der Inkongruenz ${ }^{1}$ verstanden (vgl. BiermannRatjen 2006b; Finke 2004). Somit geht es in weiterer Folge in der personzentrierten prozessualen Diagnostik darum, diese Inkongruenzen zu erfassen und differenziert zu beschreiben (vgl. Behr, 2012; Jürgens-Jahnert 2002).

„Prozessuale Diagnostik besteht darin, ausgehend von einer sorgsamen Wahrnehmung des Klienten bzw. der eigenen emotionalen Resonanz auf ihn, Verstehenshypothesen bezüglich des Klienten zu bilden und diese im therapeutischen Handeln laufend zu modifizieren" (Keil und Stumm 2018, S. 344). Sie stellt ein wichtiges Element der laufenden psychotherapeuti-

\footnotetext{
1 Unter Inkongruenz wird im personzentrierten Konzept die Nichtübereinstimmung zwischen Erfahrung und Selbstkonzept verstanden (vgl. Biermann-Ratjen 2006a); sie ist einer der zentralen Begriffe der personzentrierten Persönlichkeits- und Krankheitslehre. Genauer ausgeführt wird dieses unter dem Fokus Inkongruenz.
}

schen Arbeit dar, ist ein wesentlicher Teil des personzentrierten Behandlungskonzeptes und befindet sich permanent in Veränderung.

Fokus der personzentrierten prozessualen diagnostischen Betrachtung sind verschiedene Aspekte der Persönlichkeit, die ein ganzheitliches Verstehen der Person in ihrem Beziehungsgeflecht ermöglichen (vgl. Behr 2012). Dazu wird von Beginn an das Gewordensein der Klientin in ihrer Gesamtheit betrachtet. Die Entwicklung beziehungsweise die Stagnation des Selbstkonzeptes und daraus entstehende Inkongruenzkonstellationen stellen in diesem Zusammenhang ebenso wichtige Aspekte dar wie die Beziehungsfähigkeit der Klientin bzw. ihre Beziehungsgestaltung im Kontakt zur Therapeutin. Dabei gilt es, die subjektive Welt der Jugendlichen kennenzulernen, also ihre Erfahrungen konsequent aus ihrem inneren Bezugsrahmen heraus zu verstehen, und ihre Entwicklung nachzuvollziehen. „Als Therapeutenperson will ich verstehen, was das grundlegende Thema des Kindes ist und was es mir von seiner inneren Welt zeigt“ (Behr 2012, S. 83).

Im Folgenden wird nach einer kurzen Darstellung der Therapieeingangsdiagnostik jeder einzelne dieser vier Aspekte (s. oben kursiv hervorgehoben) hinsichtlich seiner Relevanz für den diagnostischen Prozess und für den Verlauf der Psychotherapie genauer beschrieben.

\section{Therapieeingangsdiagnostik}

In der Therapieeingangsdiagnostik werden unterschiedliche Informationen, Beobachtungen und Eindrücke zusammengetragen; sie dient der Entscheidung, ob eine Indikation zur Personzentrierten Psychotherapie gegeben ist und somit für die Jugendliche hilfreich und förderlich ist. Dies ist gegeben, wenn es sich bei den Beschwerden um eine psychische Störung handelt, die nach ICD-10 differenziert werden kann, die Jugendliche das personzentrierte Beziehungsangebot zumindest in Ansätzen wahrund annehmen kann und ein Veränderungswunsch vorhanden ist (vgl. Eckert 2006; Weinberger und Papastefanou 2008). Nach Behr (2012) dient die Eingangsdiagnostik der Klassifikation des erlebten Leidensdruckes bzw. der Symptome der Klientin, die sich phänomenologisch zeigen; sie bildet die Grundlage für eine Statusdiagnose nach ICD-10 auf der einen und für das therapeutische Vorgehen auf der anderen Seite und kann zum tieferen Verstehen der Klientin beitragen sowie für weitere Beziehungs- und Klärungsprozesse genutzt werden.

Im Anamnesegespräch werden Informationen über die bisherige Entwicklung und wichtige Erlebnisse der Jugendlichen gesammelt. Es erfolgt die Exploration der Problemsituation, bereits eingeleiteter Unterstützungsmaßnahmen, das Erfassen von bisherigen Befunden und eine Erhebung der vorhandenen Ressourcen. Die inhaltlichen Mitteilungen werden durch 
die Beobachtung der Beziehungsdynamik zwischen der Klientin und ihren Bezugspersonen ergänzt (vgl. Weinberger und Papastefanou 2008; Behr 2012; Jürgens-Jahnert 2002). Bereits zu Beginn der Therapie versucht die Personzentrierte Psychotherapeutin die unterschiedlichen emotionalen Erlebensqualitäten der beteiligten Personen - der Jugendlichen und ihren Bezugspersonen - zu erfassen und ihnen empathisch zu begegnen. Durch diese je individuelle wertfreie um Verstehen bemühte unbedingt positive Beachtung jeder einzelnen Person wird bereits die Grundlage für einen Veränderungsprozess gelegt (vgl. Rogers 1991).

Bereits im Telefongespräch entsteht ein erster Eindruck und es werden Informationen gesammelt, die für eine diagnostische Einschätzung genutzt werden können. Im Fall von Lena hat ihre Mutter angerufen und dabei einen sehr hilfsbedürftigen Eindruck gemacht. Von einer Kinder- und Jugendlichenpsychiaterin gibt es eine Zuweisung zur Psychotherapie mit der Diagnose Depression und selbstverletzendes Verhalten. Ein zeitnaher Ersttermin gemeinsam mit ihrer Tochter wird vereinbart.

Zum Erstgespräch erscheinen beide Elternteile und Lena selbst. Lena hat zu ihrer Mutter eine funktionierende Beziehung, die nicht von großer Nähe gekennzeichnet ist. Die Mutter kann kaum Äußerungen über die Innenwelt ihrer Tochter geben, durch lange Arbeitszeiten ihrerseits gibt es auch real wenig zeitliche Überschneidungen mit Lena, auch gemeinsame Aktivitäten, in denen es zu einem wechselseitigen Austausch kommen könnte, finden nicht statt. Ergänzt durch die Wahrnehmung der Beziehungsgestaltung der beiden zueinander entsteht der Eindruck, dass Mutter und Tochter eher in einer Art Wohngemeinschaft miteinander leben und weniger in einer bedeutsamen auf Gegenseitigkeit beruhenden Mutter-Tochter-Beziehung.

Die Eltern haben sich getrennt als Lena drei Jahre alt war. Lena wohnt mit ihrer alleinerziehenden Mutter; Kontakte zum Vater gibt es unregelmäßig, da dieser durch eine behandelte Alkoholerkrankung immer wieder ausfällt. Beide Eltern sind mit den Selbstverletzungen ihrer Tochter überfordert und haben keine Erklärungen für deren negative Stimmung. Bemerkbar macht sich Letztere bei Lena durch Rückzug aus ihren sozialen Kontakten, teils nur einsilbigen Antworten, stark gedrückter Stimmung und kaum außerschulischer Aktivität. Besonders die Mutter ist sehr betroffen, sie weint im Gespräch und drückt große Verzweiflung und Hilflosigkeit aus; im Gespräch sucht sie wiederholt die körperliche Nähe zu ihrer Tochter. Lena sitzt beim Gespräch dabei, möchte selbst wenig sagen und hält die meiste Zeit den Blick gesenkt.

\section{Fokus Beziehung}

Dieser ist aus folgenden drei Aspekten von Bedeutung für die personzenrierte prozessuale Diagnostik und den Psychotherapieverlauf (vgl. z. B. BiermannRatjen (2006b)):

1. Das spezifische personzentrierte Beziehungsangebot durch die Psychotherapeutin stellt die Basis für den Entwicklungsprozess dar (vgl. Rogers 1991).

2. Der zweite Aspekt ist einerseits die interaktionelle Beziehungsgestaltung der Klientin zur Psychotherapeutin, andererseits aber auch die Art und Weise der Beziehung, die die Klientin zu sich selbst aufnimmt.

3. Die Psychotherapeutin nutzt ihre Resonanz auf das Beziehungsangebot (vgl. Keil 2008).

Der Personzentrierte Ansatz ist für die Arbeit mit Jugendlichen auf Grund des speziellen Beziehungsangebotes der Psychotherapeutin in hohem Maße geeignet. Mit einem personzentrierten Beziehungsangebot, das von den therapeutischen Grundhaltungen Empathie, unbedingt positive Beachtung und Kongruenz gekennzeichnet ist (Rogers 1991) ${ }^{2}$, wendet sich die Psychotherapeutin den schnell wechselnden und oftmals widersprüchlichen Erfahrungen und Aussagen der Jugendlichen zu und ist bemüht, der Klientin aus ihrem inneren Bezugsrahmen (s.unten) heraus verstehend zu begegnen (vgl. Monden-Engelhardt 2002). „Personzentrierte Psychotherapie hilft durch die Haltung und die Gegenwärtigkeit des Therapeuten, hilft Mögliches und Gewünschtes in der Realität der therapeutischen Beziehung und in den alltäglichen Beziehungen zu erleben und zu prüfen, hilft Inkongruenzen zu erspüren und ihnen Ausdruck zu geben“ (Fehringer 2006, S. 182). Gerade jugendliche Klienten stehen der Unterstützung durch eine erwachsene Person, erst recht durch eine Psychotherapeutin, skeptisch und ambivalent gegenüber. Sie haben einerseits ein großes Bedürfnis sich mitzuteilen und ihre Probleme zu zeigen, andererseits aber sind sie auch sehr vorsichtig und selektiv in dem, was sie wirklich über sich preisgeben. Um dieser Ambivalenz und auch den ständig neuen, teils sehr intensiven Erfahrungen gerecht $\mathrm{zu}$ werden, die Jugendliche tagtäglich machen, ist eine wertfrei erlebte, tragfähige, stabile und Sicherheit gebende Beziehung Voraussetzung für den weiteren therapeutischen Prozess (vgl. Weinberger und Papastefanou 2008).

In der Therapieeingangsphase mit Lena, ist mein Fokus daher vor allem auf einen guten Kontakt zu ihr gerichtet und darauf eine vertrauensvolle Beziehung $\mathrm{zu}$ etablieren, in der wir uns ihren Schwierigkeiten zuwenden können und sie sich in ihrer Verletztheit

\footnotetext{
2 Rogers hat 1957 sechs - sowohl notwendige als auch hinreichende Bedingungen für Persönlichkeitsveränderung und Psychotherapie beschrieben: Auf Seiten der Psychotherapeutin wird eine Beziehung angeboten, die von den drei Haltungen der Kongruenz, Empathie und unbedingt positiven Beachtung gekennzeichnet ist. Auf Klientinnenseite wird ein Mindestmaß an psychologischem Kontakt, ein spürbarer Leidensdruck (Inkongruenz) und die Wahrnehmung des Beziehungsangebots der Psychotherapeutin vorausgesetzt (vgl. Rogers 1991).
} 
zeigen kann, wir aber gleichzeitig auch ihre Ressourcen würdigen.

Lenas Körperhaltung verändert sich im Zweiersetting ein wenig, sie wirkt nach wie vor zurückhaltend, scheint aber die alleinige Aufmerksamkeit zu schätzen. Sie berichtet aus ihrer Perspektive und bringt Details, die vor allem ihr emotionales Erleben beschreiben, das sie vor den Eltern nicht ansprechen wollte. Lena kann Vertrauen zu mir fassen und ich kann Erleichterung bei ihr wahrnehmen, dass sie nicht mehr alles in sich behalten muss. Sie lässt sich auf mein Beziehungsangebot ein, gleichzeitig aber ist ihr Bedürfnis nach Kontrolle hoch, was sie recht vorsichtig sein lässt.

Im Kontakt mit Lena ist zu beobachten, wie schwer es ihr fällt Augenkontakt aufzunehmen und wie hoch gleichzeitig ihr Stresslevel ist. Lenas vegetatives Nervensystem reagiert auf die hohe Anspannung stark, sie schwitzt enorm, errötet immer wieder und rote Flecken bilden sich auf ihrem Gesicht und im Halsbereich. Empathisch reagiere ich auf ihre massive Angst, indem auch ich weniger direkten Blickkontakt halte und für die erste Zeit der Therapie immer zu Beginn mit altersgerechten Gefühlskarten einsteige. Diese bieten ein sehr breites nicht eindeutig ausformuliertes Gefühlsspektrum, dass der Jugendlichen viel Raum für ihre eigene Interpretation und Darstellung des eigenen emotionalen Erlebens lassen. Gleichzeitig stellen die Karten eine Orientierungshilfe für den intrapsychischen Prozess dar und zusätzlich kann sie sich aus dem direkten Kontakt zu mir herausnehmen und sich die Zeit geben, die sie benötigt. Lena erlebt zunehmend, dass sie sich darauf verlassen kann, dass die schwarz-weißen Gefühlskarten für sie bereit liegen und sie anhand dieser über die Erfahrungen der vergangenen Woche und ihr Empfinden dazu erzählen kann. Aktives Nachfragen und konkretes Stellungbeziehen meinerseits (als Ausdruck der therapeutischen Haltung der Kongruenz bzw. Echtheit) lassen sie mich als reales, an ihr als Person interessiertes Gegenüber wahrnehmen (vgl. Behr 2012; Weinberger und Papastefanou 2008).

In der Reflexion der Beziehung zur Klientin wird die Resonanz auf das Beziehungsangebot der Jugendlichen überprüft, vor allem was am Beziehungsangebot der Jugendlichen welche Resonanz bei mir als Psychotherapeutin auslöst und worauf mich diese Resonanz im Hinblick auf ein tieferes Verstehen der Klientin hinweisen könnte (vgl. Keil 2008; Keil und Stumm 2018). Bei Lena spüre ich im ersten Kontakt ihr Bedürfnis nach Beziehung und ihr Sehnen danach, aber gleichzeitig sind ihr inkongruentes Erleben (s. unten) und die damit verbundene Ängstlichkeit ebenso wahrnehmbar. Meine Resonanz weist mich auf die Art und Weise der Beziehungsgestaltung zwischen Lena und ihren Bezugspersonen hin, in der sie früh gelernt hat ihre eigenen Wünsche und Bedürfnisse in den Hintergrund zu schieben; das Wohlergehen der Mutter, die bemüht, aber selbst recht labil ist, stand und steht im Vordergrund.

Die Art und Weise wie Lena die Beziehung zu mir gestaltet, lässt sich zu Beginn der Psychotherapie als vorsichtig beschreiben insofern, als sie mit ihren Mitteilungen zu Beginn recht zurückhaltend ist. Gleichzeitig nehme ich immer wieder auch etwas tendenziell Abwertendes wahr und spüre ihre Angst nicht ernst genommen zu werden. Den Wunsch nach Beziehung zu Gleichaltrigen kann Lena lange Zeit nur durch angepasstes Verhalten und entgegengebrachte Leistung umsetzen, was zu keiner wirklichen Befriedigung ihres Beziehungsbedürfnisses führt. Hier zeigt sich das zur Mutter erlebte Beziehungsmuster.

Im Laufe der Psychotherapie verändert sich sowohl die therapeutische Beziehung zwischen Lena und mir, als auch Lenas intrapsychische Beziehung: In der therapeutischen Beziehung macht sich das durch ihre direktere und angstfreiere Beziehungsgestaltung und durch ihre zugewandtere Körperhaltung ebenso bemerkbar wie durch zunehmend unmittelbare Äußerungen ihrer wahrgenommenen inneren Zustände, wie zum Beispiel Ärger. In der Beziehung zu sich selbst zeigt es sich in einer differenzierteren Selbstexploration und einem freundlicheren Zugewandtsein sich selbst gegenüber.

\section{Fokus Selbstkonzept}

In der personzentrierten Theorie und Praxis ist das Selbstkonzept ein zentraler Gegenstand der Betrachtung, es stellt die Grundlage für das personzentrierte Störungsmodell dar. „Das Selbstkonzept ist ein Ganzes aus der Summe und der Vernetzung aller signifikanten Erfahrungen, die das Individuum symbolisiert hat. Es besteht aus Vorstellungen, Bildern, Empfindungen, Gefühlen, Gedanken, Worten, Sätzen und den dazugehörigen Werten“ (Heinerth 2003, S. 278). Symbolisiert werden bzw. Eingang ins Selbstkonzept finden nach Biermann-Ratjen (2006a) nur solche Erfahrungen, die von einer kongruenten Bezugsperson unbedingt positiv beachtet und empathisch verstanden wurden.

Die Beachtung der Entwicklung des Selbstkonzeptes ist für die personzentrierte Diagnostik insofern relevant, als in jeder einzelnen Phase der Selbstkonzeptentwicklung je nach Erfahrung des Wahr- und Angenommenwerdens spezifische Störungen auftreten können. Die Entwicklung des Selbstkonzeptes, die immer ein interaktioneller Prozess ist, vollzieht sich nach der personzentrierten Entwicklungslehre in drei Phasen (vgl. Biermann-Ratjen 2006a, 2006b; Biermann-Ratjen et al. 2003) und beginnt bereits in der frühen Mutter-Kind-Interaktion in Form der Einstimmung der Mutter auf die vor allem körperlich ausgedrückten Bedürfnisse des Kindes. Im Zentrum steht die feinfühlige Beantwortung der ausgedrückten Grundbedürfnisse des Kindes durch die Mutter, wodurch das Kind eine sichere und haltende Beziehung erfährt; hier zeigen sich Parallelen zur Bindungs- 
theorie (vgl. Jürgens-Jahnert 2002; Biermann-Ratjen 2006a). Ziel in dieser ersten Phase ist es, aus affektiven Erfahrungen Selbsterfahrungen zu entwickeln, die als solche Teile des Selbstkonzeptes werden. Kinder, die in dieser Phase keine feinfühlige Regulation durch die Mutter erfahren, erleben ein hohes Maß an Anspannung und Angst; das Ergebnis ist nach BiermannRatjen (2006b) ein labiles Selbstkonzept, in dem nur wenige bis keine dieser affektiven Erfahrungen als Selbsterfahrungen abgebildet sind; das Individuum fühlt sich von (fast) jeder affektiven Erfahrung in seiner Existenz bedroht, Vernichtungsängste werden erlebt (vgl. ebd.).

In der zweiten Phase der Selbstkonzeptentwicklung taucht das subjektive Selbst auf. Das bedeutet, dass sich das Kind, obwohl immer noch in einer präsymbolischen Phase, selbst als Akteurin erlebt, seine Handlungen und Affekte wahrnimmt und das Bedürfnis nach positiver Selbstbeachtung wird erfahrbar. „Personen, deren Entwicklung in der zweiten Phase stagniert, erleben Inkongruenz weniger als Angst vor dem Zusammenbruch ihres Selbst, dem Chaos oder der Vernichtung. Sie erleben in ihrer Inkongruenz Angst, absolut böse und wertlos zu sein (und deshalb verlassen $\mathrm{zu}$ werden bzw. mutterseelenallein $\mathrm{zu}$ sein)“ (Biermann-Ratjen 2006b, S. 109). Das Selbstkonzept ist somit durch Erfahrungen bedroht, die die Selbstachtung in Frage stellen.

Die Möglichkeit des verbalen Ausdrucks eröffnet in der dritten Phase die Mitteilung des eigenen Erlebens und ist ein lebenslanger Prozess, in dem es immer wieder um die Herstellung von Kongruenz zwischen Selbstkonzept und Erfahrung geht. In der dritten Phase kann das Kind „... sein Interesse darauf richten, was es sein und werden kann - in der eigenen Wahrnehmung und in den Augen anderer ..." (Biermann-Ratjen et al. 2003, S. 92). Stagnation in dieser Phase der Selbstkonzeptentwicklung bedeutet Ängste $\mathrm{zu}$ verspüren, in seinem Sosein nicht $\mathrm{zu}$ genügen, sondern von einem Gegenüber anders gedacht oder gewollt zu sein.

In der Pubertät kommt es zu einer Umstrukturierung des Selbstkonzeptes und die bereits erwähnten vielfältigen Erfahrungen, die Veränderungen auf physischer, sozialer, aber auch psychischer Ebene darstellen, werden immer wieder dahingehend überprüft, ob sie in das bestehende Selbstkonzept passen und somit darin aufgenommen werden können (vgl. Weinberger und Papastefanou 2008). Ist dies nicht möglich, wird die Erfahrung abgewehrt und kann nur in verzerrter Form Eingang ins Selbstkonzept finden bzw. muss komplett verleugnet werden; der Zustand, der dadurch ausgelöst wird, ist von Angst und Anspannung gekennzeichnet und wird als Inkongruenz (s. unten) bezeichnet (vgl. Biermann-Ratjen 2006a, 2006b; Biermann-Ratjen et al. 2003; Rogers 1987).

Lenas Selbstkonzeptentwicklung stagniert vor allem in der zweiten und dritten Phase. Sie drückt dies zunächst durch in der Therapie entstehende Bilder aus und in einer späteren Therapiephase auch verbal. Im prozessualen diagnostischen Prozess stellen wir immer wieder fest, welche Erfahrungen gerade von Lena wahrgenommen werden und wie sie diese in Bezug zu ihrem Selbstkonzept erlebt. Dementsprechend legt sie ihre Wünsche und Ziele für den weiteren Prozess fest, z. B. selbstsicherer werden, gegenüber anderen Stellung beziehen können, in Beziehung $\mathrm{zu}$ anderen die eigenen Bedürfnisse trotzdem leben.

\section{Fokus Inkongruenz}

Inkongruenz „... bezeichnet die konflikthaltige innerpsychische Diskrepanz, die besteht, wenn Erfahrungen vom Individuum nicht als Selbsterfahrungen wahr- und angenommen werden, weil ihre Gewahrwerdung abgewehrt wird, und/oder nur in verzerrter und entstellter Form symbolisiert werden können“ (Keil 2003, S. 175).

Im Zuge des diagnostischen Prozesses wird versucht, aus dem inneren Bezugsrahmen (s.unten) der Jugendlichen heraus zu verstehen, welche Erfahrungen aus dem lebensgeschichtlichen Gewordensein ein inkongruentes Verarbeiten notwendig machen. Jene Erfahrungen, die für das Selbstkonzept bedrohlich sind und dadurch eine Inkongruenz bewirken, gilt es im diagnostischen Prozess ausfindig zu machen. Die Aufgabe der Psychotherapeutin ist es, mit Hilfe einer Verstehenshypothese, die im Therapieverlauf entsteht, der Jugendlichen in der unmittelbaren Beziehung zu begegnen und diese als Verstehensangebot zur Verfügung zu stellen. Ist diese nicht passend oder auch noch zu bedrohlich für die Klientin, nimmt die Psychotherapeutin die Reaktion der Jugendlichen durch ihre Resonanz wahr und diese Wahrnehmung fließt wiederum in den weiteren Verstehensprozess ein (vgl. Keil und Stumm 2018).

$\mathrm{Zu}$ Beginn der Therapie wird von Lena vorwiegend Traurigkeit und ein Empfinden von Resignation und Enttäuschung wahrgenommen, es ist ihr somit nur ein Teil ihres Erlebens zugänglich. Ihr Bedürfnis nach Beziehung zu Gleichaltrigen und ihr Wunsch nach Freundschaft ist groß, die damit im Zusammenhang stehenden Ängste vor Zurückweisung und ihrer eigenen Wut beziehungsweise ihrem Ärger darüber, nicht angenommen $\mathrm{zu}$ werden und sich benutzt $\mathrm{zu}$ fühlen, bedrohen jedoch ihr Selbstkonzept und können nicht bewusst wahrgenommen werden. Da sie selbst in der Schule sehr gut ist, wird sie oft von anderen Schülern um Unterstützung angefragt, die sie gerne gibt (das entspricht ihrem eigenen Bild von sich, also ihrem Selbstkonzept). Dabei ist sie aber immer wieder mit dem Abbruch der Treffen konfrontiert. Bei Grenzüberschreitungen ist es für Lena schwierig sich selbst $\mathrm{zu}$ verteidigen und $\mathrm{zu}$ behaupten. In ihr auftauchende ärgerliche Gefühle darüber stellen eine Bedrohung für ihr Selbstkonzept dar. Das Erleben des eigentlichen Gefühls, nämlich Ärger, muss abgewehrt werden und infolge der inkongruenten 
Verarbeitung kann sie wiederum nur Enttäuschung und Wertlosigkeit (als verzerrtes Abbild ihres Ärgers im Selbstkonzept) spüren und sich aus den sozialen Kontakten zurückziehen.

Auch die Angst vor der eigenen Verletzbarkeit und Schwäche wird inkongruent verarbeitet (s. oben). Diese Angst ist ein Schutz vor der Erfahrung, selbst die Bedürftige und Hilfesuchende zu sein und auf die Unterstützung von anderen angewiesen zu sein, wodurch dieser Teil ihrer Persönlichkeit ausgegrenzt wird.

Lena benötigt oftmalige Zuwendung gegenüber allen Aspekten ihres Erlebens, insbesondere jenen, die nicht im Selbstkonzept integriert sind und in denen sie sich selbst (noch) nicht annehmen kann, und ein immer wieder neu Verstehen, warum ein teilweise inkongruentes Verarbeiten noch notwendig ist.

Den beschriebenen Inkongruenzen liegen die nachfolgend lebensgeschichtlichen Zusammenhänge zu Grunde. So war die Mutter oftmals nicht in der Lage Lena kongruent $\mathrm{zu}$ begegnen und hat versucht ihre eigene Gekränktheit und teilweise Überforderung durch die familiäre Situation nicht zu zeigen; sie war in ihrem Affektausdruck gegenüber Lena gehemmt. Lena war in vielen Situationen ihren Gefühlen selbst überlassen und damit überfordert. Wut und Verzweiflung, die bei ihr auftauchten, konnten von der Mutter nicht wahrgenommen und verstanden werden und somit nicht in ihr Selbstkonzept integriert werden; stattdessen stellen Depression und Resignation einen Ausdruck verzerrter Abbildung im Selbstkonzept dar. Früh hat sich in ihr die Angst manifestiert ihre Mutter zu verlieren und alleine zu sein, wodurch Lena gelernt hat, ihre eigenen Bedürfnisse zurückzustecken und ärgerliche Gefühle über die Nichtbeachtung kaum mehr wahrzunehmen.

\section{Fokus Innerer Bezugsrahmen}

„Dieser Begriff umfaßt die gesamte Breite von Empfindungen, Wahrnehmungen, Bedeutungen und Erinnerungen, die der Gewahrwerdung zugänglich sind. Der innere Bezugsrahmen ist die subjektive Welt des Individuums“ (Rogers 1987, S. 37). Der innere Bezugsrahmen der Jugendlichen ist quasi der Ausgangspunkt, aus dem sich sowohl das Selbstkonzept, als auch die Hypothesen über die Inkongruenz erschließt.

Diagnostik als prozessorientiertes Geschehen nimmt die Perspektive der Jugendlichen als bedeutenden Faktor mit auf in den Prozess. Durch empathisches Einfühlen, aber auch konkretes Nachfragen und kreative Ausdrucksangebote versucht die Therapeutin sich dieser inneren Welt anzunähern und das subjektive Verstehen zu unterstützen. In der Arbeit mit Jugendlichen ist es darüber hinaus wichtig, sich auch mit deren Lebenswelten und Interessen insgesamt zu beschäftigen und Wissen darüber anzueignen.

So möchte ich abschließend den Prozess von Lena unter dem Fokus des inneren Bezugsrahmens, der gleichzeitig Ausgangspunkt für den gesamten Prozess ist, zusammenfassend darstellen. Lenas Erlebensradius war zu Beginn der Therapie sehr eigeschränkt. Lena hatte von sich das Bild (Selbstkonzept), keinen Wert zu haben, nicht wichtig zu sein und fühlte sich auch nicht attraktiv. Darum zog sie sich immer mehr aus den wenigen sozialen Kontakten, die sie hatte, zurück und äußerte suizidale Absichten (Teil der Inkongruenz). Lenas Ressourcen - ihre Beziehungsfähigkeit, ihr Mut sich auf den Prozess einzulassen und ihre Neugierde auf das eigene Erleben - ermöglichen den therapeutischen Prozess, den Lena selbst wiederum als stützend und hilfreich wahr- und annehmen kann.

Lenas Stimmung ist in der ersten Zeit der Therapie sehr negativ, ihre sozialen Kontakte sind eingeschränkt und mit hohen Erwartungen ihrerseits überlagert. Die Wahrnehmung eigener Bedürfnisse fällt ihr sehr schwer, die Formulierung beziehungsweise Umsetzung dieser gelingt quasi gar nicht. Es bereitet Lena große Sorge, wie sie von ihrem Umfeld wahrgenommen wird, in dem sie tendenziell Abneigung und Abwertung spürt. Sie formuliert immer wieder die Angst, ihrem Umfeld nicht zu entsprechen und Abwertung zu erfahren.

Im Laufe der Psychotherapie macht Lena zunehmend mutige Schritte, es gelingt ihr eigene Stärken aber auch Schwächen zu formulieren und ihre eigenen Bedürfnisse mehr in den Fokus zu rücken. Ein breiteres Spektrum an unterschiedlichen Erfahrungen darf gemacht werden und wird in ihr Selbstkonzept integriert. Die teilweise rigiden Bilder und Verarbeitungsmodi, die zu Beginn der Therapie vorherrschten und sich in der prozessualen Diagnostik immer wieder zeigten, nimmt Lena selbst offener wahr. Lenas Persönlichkeit ist insgesamt offener für neue Erfahrungen und flexibler im Umgang damit geworden.

\section{Zusammenfassung}

Die Personzentrierte Psychotherapie ist entwicklungsorientiert; ihr Ziel ist es, die Inkongruenz zwischen Erfahrung und Selbstkonzept zu reduzieren, beziehungsweise aufzuheben und somit eine Weiterentwicklung des Selbstkonzeptes zu ermöglichen. Das diagnostische Erfassen der oben beschrieben Aspekte dient als Grundlage für den therapeutischen Prozess.

Die personzentrierte Diagnostik ist neben der Eingangsdiagnostik, zu der auch die Diagnostik nach ICD-10 gehört, vor allem eine prozessuale; sie dient der Überprüfung, wo der therapeutische Prozess gerade steht und welche Ziele, die sich im Therapieverlauf mitverändern, die Klientin verfolgen möchte. Die Ziele werden im therapeutischen Prozess immer wieder konkret ausgesprochen und gegebenenfalls neu definiert. „Wenn nun die Therapie in einem fortlaufenden Explorationsprozess besteht, kann sie zugleich auch als diagnostischer Vorgang betrachtet werden (Therapie= Diagnose, ,Theragnose') " (Keil und Stumm 2018, S. 336). 
Für eine umfassende personzentrierte Diagnostik benötigt die Personzentrierte Psychotherapeutin eine Vielzahl an Fertigkeiten und Wissen aus unterschiedlichen Feldern. Neben fundiertem entwicklungspsychologischem Wissen und der Bedeutung der Bindungsmuster ist auch eine ständige Auseinandersetzung mit der eigenen inneren Welt und den aktuellen Lebenswelten von Jugendlichen notwendig. Zusätzlich ist es wichtig störungsspezifisches Wissen in die Verstehenshypothesen mit einfließen zu lassen und somit für den diagnostischen und therapeutischen Prozess zu nutzen. Abschließen möchte ich mit folgenden Worten von Behr (2012, S. 10): „Sowohl die Kenntnis störungsspezifischen Wissens um Diagnostik, Indikation und Behandlung sind bedeutend, als auch die Option, die Person als einzigartig in ihrem Sein zu verstehen und ihr bei der Organisation ihrer Erfahrungen und Problemlösungen zu helfen, ihren individuellen Weg zu finden.“

Interessenkonflikt $M$. Brückl gibt an, dass kein Interessenkonflikt besteht.

Open Access Dieser Artikel wird unter der Creative Commons Namensnennung 4.0 International Lizenz veröffentlicht, welche die Nutzung, Vervielfältigung, Bearbeitung, Verbreitung und Wiedergabe in jeglichem Medium und Format erlaubt, sofern Sie den/die ursprünglichen Autor(en) und die Quelle ordnungsgemäß nennen, einen Link zur Creative Commons Lizenz beifügen und angeben, ob Änderungen vorgenommen wurden.

Die in diesem Artikel enthaltenen Bilder und sonstiges Drittmaterial unterliegen ebenfalls der genannten Creative Commons Lizenz, sofern sich aus der Abbildungslegende nichts anderes ergibt. Sofern das betreffende Material nicht unter der genannten Creative Commons Lizenz steht und die betreffende Handlung nicht nach gesetzlichen Vorschriften erlaubt ist, ist für die oben aufgeführten Weiterverwendungen des Materials die Einwilligung des jeweiligen Rechteinhabers einzuholen.

Weitere Details zur Lizenz entnehmen Sie bitte der Lizenzinformation auf http://creativecommons.org/licenses/by/4. $0 /$ deed.de.

\section{Literatur}

\section{Verwendete Literatur}

Behr, M. (2012). Störungsspezifische Handlungsleitlinien in der Kinder- und Jugendlichenpsychotherapie-Eine Herausforderung für ganzheitlich und störungsbezogen ausgerichtete Verfahren. PERSON, 16(1), 5-12.

Behr, M., Hölldampf, D., \& Hüsson, D. (Hrsg.). (2009). Psychotherapie mit Kindern und Jugendlichen. Personzentrierte Methoden und interaktionelle Behandlungskonzepte. Göttingen: Hogrefe.

Behr, M., Hölldampf, D., \& Steiger, T. (2012). Themenheft Kinder- und Jugendlichenpsychotherapie. PERSON, 16(1), 5-83.

Biermann-Ratjen, E.-M. (2006a). Klientenzentrierte Entwicklungslehre. In J.Eckert, E.-M. Biermann-Ratjen \&D. Höger (Hrsg.), Gesprächspsychotherapie. Lehrbuch für die Praxis (S.73-91). Heidelberg: Springer.
Biermann-Ratjen, E.-M. (2006b). Krankheitslehre der Gesprächspsychotherapie. In J. Eckert, E.-M. BiermannRatjen \&D.Höger (Hrsg.), Gesprächspsychotherapie. Lehrbuch für die Praxis (S.93-116). Heidelberg: Springer.

Biermann-Ratjen, E.-M., Eckert, J., \& Schwartz, H. (2003). Gesprächspsychotherapie. Verändern durch verstehen. Stuttgart: Kohlhammer.

Eckert, J. (2006). Indikationsstellung. In J. Eckert, E.-M. Biermann-Ratjen \& D. Höger (Hrsg.), Gesprächspsychotherapie. Lehrbuch für die Praxis (S. 219-266). Heidelberg: Springer.

Fehringer, C. (2006). Ja, so ist die Jugend heute, schrecklich sind die jungen Leute. (W. Busch) Personzentrierte Psychotherapie mit Jugendlichen. PERSON, 10(2), 176-186.

Finke, J. (2004). Gesprächspsychotherapie. Grundlagen und spezifische Anwendungen (3. Aufl.). Stuttgart: Thieme.

Heinerth, K. (2003). Selbst, Selbstkonzept. In G. Stumm, J. Wiltschko \& W.W. Keil (Hrsg.), Grundbegriffe der Personzentrierten und Focusing-orientierten Psychotherapie und Beratung(S.278-280). Stuttgart: Klett-Cotta.

Jürgens-Jahnert, S. ( (2002). Therapieeinleitung und Diagnostik in der personzentrierten Psychotherapie mit Kindern und Jugendlichen: einige theoretische Überlegungen und praktischeAnregungen. In C. Boeck-Singelmann, B. Ehlers, T. Hensel, F. Kemper \& C. Monden-Engelhardt (Hrsg.), Personzentrierte Psychotherapie mit Kindern und Jugendlichen 2. Aufl. (Bd. 2, S. 257-285). Göttingen: Hogrefe.

Keil, W.W. (2003). Inkongruenz. In G. Stumm, J. Wiltschkob \& W.W. Keil (Hrsg.), Grundbegriffe der Personzentrierten und Focusing-orientierten Psychotherapie und Beratung (S. 175-177). Stuttgart: Klett-Cotta.

Keil, W.W. (2008). Die prozessuale Diagnostik in der Klientenzentrierten Psychotherapie. In M. Tuczai, G. Stumm, D. Kimbacher \& N. Nemeskeri (Hrsg.), Offenheit und Vielfalt. Personzentrierte Psychotherapie: Grundlagen, Ansätze, Anwendungen (S. 167-184). Wien: Krammer.

Keil, W. W., \& Stumm, G. (2018). Diagnostik und Indikation. In G.Stumm\&W.W.Keil(Hrsg.), Praxis der Personzentrierten Psychotherapie (S.329-344). Wien: Springer.

Monden-Engelhardt, C. (2002). In C. Boeck-Singelmann, B. Ehlers, T. Hensel, F. Kemper \& C. Monden-Engelhardt (Hrsg.), Personzentrierte Psychotherapie mit Kindern und Jugendlichen 2.Aufl. Bd.2. Göttingen: Hogrefe.

Reisel, B. (2014). The clinical Treatment of the Problem Child. Carl Rogers als Kindertherapeut. In M. Behr, D. Hüsson, D. Nuding \& C. Wakolbinger (Hrsg.), Psychotherapie und Beratung bei Kindern, Jugendlichen und Familien. Personzentrierte Beiträge aus zwei Jahrzehnten. Wien: Facultas.

Rogers, C. R. (1987). Eine Theorie der Psychotherapie, der Persönlichkeit und der zwischenmenschlichen Beziehungen. Köln: GwG.

Rogers, C.R. (1991). Die notwendigen und hinreichenden Bedingungen für Persönlichkeitsveränderung durch Psychotherapie. In M. Werkmeister \& P. F. Schmid (Hrsg.), Person-zentriert: Grundlagen von Theorie und Praxis. Mit einem kommentierten Beratungsgespräch von Carl Rogers (1.Aufl. S. 165-184). Mainz: Grünewald.

Rogers, C.R. (2003). Die klientenzentrierte Gesprächspsychotherapie. Client-Centred Therapy. Frankfurt am Main: Fischer.

Weinberger, S., \& Papastefanou, C. (2008). Wege durchs Labyrinth. Personzentrierte Beratung und Psychotherapie mit Jugendlichen. Weinheimund München: Juventa. 


\section{Weiterführende Literatur}

Rogers, C.R. (1939). The clinical treatment of the problem child. Boston: Houghton Mifflin.

Hinweis des Verlags Der Verlag bleibt in Hinblick auf geografische Zuordnungen und Gebietsbezeichnungen in veröffentlichten Karten und Institutsadressen neutral.
Marieluise Brückl, Sonder- und Heilpädagogin, Personzentrierte Psychotherapeutin, Kinder und Jugendlichenpsychotherapie, Supervision, Psychotherapeutische Arbeit in freier Praxis mit Erwachsenen, Jugendlichen und Kindern, Leiterin der Weiterbildung „Personzentrierte Psychotherapie mit Kindern und Jugendlichen" der Sektion FORUM gemeinsam mit derVRP. 